Leishmania causante de LTA no solo para propósitos taxonómicos y epidemiológicos, sino porque la eficacia de la respuesta a la vacuna pentavalente depende de la especie del parásito circulante.

El objetivo de este estudio fue describir las características clínicas, diagnósticas y epidemiológicas de la LTA en Acre, un estado de la Amazonia occidental brasileña, de acuerdo con la especie de Leishmania causante de la enfermedad.

El estudio se llevó a cabo entre marzo y septiembre de 2002 en el municipio de Rio Branco, donde se ha presentado $25 \%$ de los casos de LTA notificados por las autoridades sanitarias en los últimos 3 años. La muestra de conveniencia estuvo compuesta por 50 personas mayores de 6 años que presentaron lesiones cutáneas características de la leishmaniasis con más de dos semanas de evolución y sin afectación de las mucosas. Se emplearon técnicas convencionales y de biología molecular para confirmar el diagnóstico parasitológico e identificar la especie causante de la enfermedad.

Las características clínicas de la leishmaniasis cutánea observadas en este estudio fueron similares a las de otras regiones de Brasil, donde las especies pertenecientes al subgénero Leishmania (Viannia) son las responsables de la mayoría de los casos de LTA. El predominio de L. (V.) braziliensis y L. (V.) guyanensis en este estudio refleja la epidemiología de LTA en Brasil, ya que estas dos especies son las más frecuentemente descritas en las diferentes regiones endémicas del país. No se encontraron signos clínicos asociados con alguna especie en particular, lo que confirma el hecho de que es prácticamente imposible identificar la especie del parásito a partir de los signos y síntomas clínicos del paciente.

El creciente número de casos de LTA en el estado de Acre puede explicarse por el mejoramiento del sistema de vigilancia, las migraciones laborales de personas susceptibles hacia zonas de mayor transmisión, la afectación del equilibrio en los reservorios naturales debido a la deforestación y la explotación de los productos forestales, como frutas, caucho y madera.

Estos resultados muestran la gran diversidad del género Leishmania en el estado de Acre, donde circulan al menos tres especies y varios híbridos putativos. La diversidad de genotipos fue aun mayor, pues se describieron por primera vez nueve zimodemos, siete de ellos pertenecientes al complejo de $L$. $(V$.) braziliensis, lo que indica que hay una considerable diversidad aun dentro de una misma especie de este parásito.

Según estos resultados, se puede esperar que los ciclos de transmisión de este parásito sean también muy heterogéneos y que en ese ciclo participen varios vectores flebótomos y diversos organismos como reservorios. Por estas razones, las medidas de control basadas en el comportamiento del vector son sumamente complejas y evitar la transmisión puede ser muy difícil en regiones en las que hay políticas que respaldan la permanencia de personas susceptibles en zonas selváticas para realizar diversas actividades. (Tojal da Silva AC, Cupolillo E, Volpini AC, Almeida R, Sierra Romero GA. Species diversity causing human cutaneous leishmaniasis in Rio Branco, state of Acre, Brazil. Trop Med Int Health. 2006;11:1388-98.)

\section{Reflexiones sobre el desarrollo de capacidades para la promoción de salud en Perú}

En los últimos años, el desarrollo de capacidades ha estado en la agenda de los gobiernos donantes de recursos y de las agencias de desarrollo. El término "desarrollo de capacidades" se utiliza para denominar el proceso de creación y fortalecimiento de competencias y habilidades, tanto en instituciones como en personas individuales, que les facilite lograr el cumplimiento de sus objetivos.

En este artículo se analiza una intervención realizada en Perú para desarrollar capacidades dirigidas a la promoción de salud. La premisa básica de esa intervención fue que el fortalecimiento de la capacidad de las personas y de las instituciones para promover la salud contribuiría a mejorar las condiciones de salud y los hábitos saludables en todo el país.

A pesar de que en este trabajo no se hace un análisis final del impacto de esta intervención, ya que no se han evaluado formalmente las actividades realizadas, los resultados expuestos permiten extraer algunas lecciones que pueden ser de utilidad en el diseño de intervenciones dirigidas a desarrollar capacidades, especialmente para la promoción de salud.

La intervención se diseñó y llevó a cabo por el Proyecto Cambio entre 2001 y 2004 con la participación de cuatro universidades privadas (la Pontificia Universidad Católica, la Universidad Cayetano Heredia, la Universidad de Lima y la Universidad del Pacífico) y dos organizaciones no gubernamentales (ONG) peruanas con experiencia reconocida en la promoción de salud en la zona: Apoyo a Programas de Población (APROPO) y PRISMA. Se organizaron mesas redondas regionales en ocho regiones del país, talleres para los profesores, el personal de las ONG y el Ministerio de Salud de Perú, entrenamientos en la cobertura de noticias sobre salud para estudiantes y periodistas, un programa de becas dirigido a la comunicación y promoción de salud y un programa de prácticas estudiantiles en empresas.

Los resultados de esta intervención demuestran que el compromiso a largo plazo de los donantes $\mathrm{y}$ de las agencias de financiamiento es insuficiente 
para resolver satisfactoriamente los problemas que provoca la falta de dedicación y empeño en proyectos de desarrollo de capacidades. El fortalecimiento de las capacidades del gobierno central y de las autoridades regionales parece enfrentar problemas permanentes, como los trámites burocráticos, el cambio constante de personal, la sobrecarga de trabajo, los bajos salarios y las presiones políticas para abandonar los planes promovidos por la anterior administración. La asociación entre universidades, ONG y organizaciones privadas de carácter voluntario puede tener menos obstáculos, pero no está exenta de retos.

Los proyectos de desarrollo de capacidades no pueden desconocer estas condiciones a la hora de diseñar intervenciones, especialmente en países en desarrollo como Perú, donde el sistema público de salud, a pesar de sus limitaciones, hace un esfuerzo para ofrecer sus servicios a la mayoría de la población. Los proyectos de desarrollo de capacidades pueden orientar su trabajo hacia organizaciones de la sociedad civil, pero si no promueven el fortalecimiento de capacidades en las instancias de gobierno, sus acciones tendrán poco impacto en los programas de salud y, finalmente, en la población.

Es necesario promover un pensamiento creativo para reconceptualizar los programas de entrenamiento, una actividad obligada en cualquier proyecto de desarrollo de capacidades. Estos programas deben satisfacer las demandas institucionales y cumplir con las cuotas de participación en los entrenamientos. Sin embargo, el desarrollo de capacidades no es sinónimo de entrenamiento, especialmente cuando los sistemas de salud padecen un constante robo de cerebros, la moral es baja entre el personal y faltan incentivos institucionales para mejorar el desempeño laboral. En tales circunstancias, el impacto de las actividades de entrenamiento en la promoción de salud y en la salud pública puede durar poco.

El fortalecimiento de las capacidades implica establecer vínculos de colaboración entre las instituciones, estipulando claramente los términos de colaboración y garantizando que todas las partes se sientan igualmente comprometidas con los mismos resultados. Sin estas premisas, la responsabilidad compartida sería muy frágil y la asociación y las metas podrían ser efímeras. (Waisbord S. When training is insufficient: reflections on capacity development in health promotion in Peru. Health Prom Int. 2006;21(3):230-7.)

\section{Seroprevalencia y factores de riesgo de la infección por el VIH en trabajadoras sexuales de América del Sur}

La prevalencia de la infección por el virus de la inmunodeficiencia humana tipo 1 (VIH-1) varía considerablemente entre las poblaciones de trabajadoras sexuales de diferentes partes del mundo. Las mayores prevalencias se han encontrado en los países de África subsahariana (de 0,2\% a 60,5\%), seguidos de los del sur y el sudeste de Asia (hasta 26\%). En América Latina y el Caribe, según los datos disponibles, las tasas son menores (hasta $14,0 \%$ ) y la transmisión de la infección por VIH se concentra en otros grupos de alto riesgo, como los hombres que tienen sexo con hombres y los adictos a drogas inyectadas. Sin embargo, esta información es fragmentada e insuficiente.

En este artículo se resumen los datos de varios estudios transversales que en conjunto abarcaron 13600 trabajadoras sexuales de 32 ciudades de América del Sur representativas de la región andina (Bolivia: Santa Cruz; Colombia: Bogotá; Ecuador: Quito y Guayaquil; Perú: Lima, Iquitos, Pucallpa, Trujillo, Arequipa, Tacna y Sullana; y Venezuela: Isla Margarita) y del Cono Sur (Argentina: Buenos Aires y otras siete ciudades; Chile: Santiago; Paraguay: Asunción y otras cuatro ciudades; y Uruguay: Montevideo y cinco ciudades fronterizas con Brasil). El estudio se llevó a cabo entre 1999 y 2002 mediante cuestionarios anónimos aplicados en burdeles, salones de masaje, hoteles y calles. Se tomaron muestras de sangre para la detección serológica de la infección por VIH mediante pruebas inmunoenzimáticas del tipo ELISA y su confirmación se realizó mediante pruebas de western blot. Todas las participantes tenían 18 años de edad o más y aceptaron tomar parte en el estudio. Se calculó la razón de posibilidades ajustada por la edad (RPA).

La prevalencia general fue de 1,2\% y varió de 0,0\% (en Venezuela y Chile) a 4,5\% (en Argentina), con valores intermedios en los restantes países (Bolivia: 0,4\%, Colombia: 0,8\%, Uruguay: 0,8\%, Perú: 1,0\%, Ecuador: 1,8\% y Paraguay: 2,6\%). En conjunto, la prevalencia de VIH fue menor en los países de la región andina que en los del Cono Sur. Los factores de predicción de esta infección en la región andina fueron los antecedentes de infección de transmisión sexual (RPA $=3,8-8,3)$ y la vinculación al comercio sexual por más de 10 años (RPA $=2,2-24,8)$. El contacto sexual con más de tres clientes $(R P A=5,0)$, las relaciones sexuales con extranjeros $(\mathrm{RPA}=6,9 \%)$, el uso de drogas ilegales (RPA $=3,2$ ) y el consumo de marihuana ( $R P A=8,2)$ estuvieron asociados con la infección por VIH en los países del Cono Sur.

Estos resultados confirman que la prevalencia de la infección por el VIH es baja en las trabajadoras sexuales de América del Sur, especialmente en los países de la región andina. Se observó un aumento en la prevalencia en Argentina, Paraguay y Uruguay. En Argentina, 65\% de las mujeres infectadas se encontraron en la zona metropolitana de Buenos Aires. 